Wilfrid Laurier University

Scholars Commons @ Laurier

Physics and Computer Science Faculty

Publications

Physics and Computer Science

$5-2007$

\title{
Low-Cost High-Sensitivity Strain and Temperature Sensing Using Graded-Index Multimode Fibers
}

Yu Liu

Wilfrid Laurier University

Li Wei

Wilfrid Laurier University, Iwei@wlu.ca

Follow this and additional works at: https://scholars.wlu.ca/phys_faculty

\section{Recommended Citation}

Liu, Yu and Wei, Li, "Low-Cost High-Sensitivity Strain and Temperature Sensing Using Graded-Index Multimode Fibers" (2007). Physics and Computer Science Faculty Publications. 18.

https://scholars.wlu.ca/phys_faculty/18

This Article is brought to you for free and open access by the Physics and Computer Science at Scholars Commons @ Laurier. It has been accepted for inclusion in Physics and Computer Science Faculty Publications by an authorized administrator of Scholars Commons @ Laurier. For more information, please contact scholarscommons@wlu.ca. 


\title{
Low-cost high-sensitivity strain and temperature sensing using graded-index multimode fibers
}

\author{
Yu Liu and Li Wei
}

\begin{abstract}
We report a low-loss, low-cost high-sensitivity all-fiber strain and temperature sensor based on mode interference in graded-index multimode fibers. Blueshifts with strain and temperature sensitivities of $18.6 \mathrm{pm} /$ microstrain and $58.5 \mathrm{pm} /{ }^{\circ} \mathrm{C}$ have been observed. Experimental results show that smaller core diameter graded-index fibers display greater strain-induced peak wavelength shifts than larger core diameter fibers. (C) 2007 Optical Society of America

OCIS codes: $\quad 060.2310,060.2370$.
\end{abstract}

\section{Introduction}

Fiber-optic sensors have been widely used in a variety of sensing because of their inherent advantages. They are lightweight, compact, resistant to electromagnetic interference, and able to be embedded into other structures. Based on the principle of the operation, fiber-optic sensors can be classified into intensity based, spectrally based, and interferometric fiber-optic sensors, all of which may be implemented with very simple structures in the form of optical fibers or fiber Bragg gratings (FBGs). For example, intensity-based fiber-optic sensors employed optical fibers with a certain portion of modified cladding material $^{1}$ for chemical and biochemical sensing, while single-mode $\mathrm{FBGs}^{2}$ and long-period fiber gratings $^{3}$ (LPFGs) are commonly used as spectrally based fiber-optic sensors in strain and temperature sensing. Interferometric fiber-optic sensors are of great interest because of their high sensitivities. Recently, allfiber Mach-Zehnder interferometric sensors ${ }^{4-8}$ based on mode interference have increasingly aroused research interests because they are compact and easy to fabricate. For example, Wang and Farrell ${ }^{5}$ proposed an all-fiber multimode-interference-based refractometer for chemical sensor, and Mehta et al. ${ }^{6}$ investigated multimode interference-based Mach-

The authors are with the Department of Physics and Computer Science, Wilfrid Laurier University, Waterloo, Ontario N2L 3C5, Canada. Y. Liu's e-mail address is georgel@uhnres.utoronto.ca.

Received 22 August 2006; revised 18 December 2006; accepted 19 December 2006; posted 20 December 2006 (Doc. ID 74308); published 9 April 2007.

0003-6935/07/132516-04\$15.00/0

(C) 2007 Optical Society of America
Zehnder interferometers (MZIs) for displacement sensors. Temperature sensors were also demonstrated based on all-fiber MZIs, but special fibers, e.g, liquidcrystal-clad fibers ${ }^{7}$ or two-mode fibers, ${ }^{8}$ were employed, and the devices had relatively high insertion loss.

Here we report a low-cost, low-insertion loss and high sensitivity MZI based on mode interference in a standard graded-index (GI) multimode fiber for both strain and temperature sensing. Such a sensor can be formed by splicing two single-mode fibers to both sides of a GI multimode fiber, where the two splicing points act as two mode couplers. One splicing point couples the fundamental core mode from the single-mode fiber to the different modes in the multimode fiber, and the other recouples them back to the single-mode fiber. Experiments show only two main modes are involved in the interference. Blueshifts (i.e., wavelength shifts to the shorter wavelength side) were observed with sensitivities of $18.6 \mathrm{pm} /$ microstrain and $58.5 \mathrm{pm} /{ }^{\circ} \mathrm{C}$ for applied strain and temperature changes, respectively, whereas redshifts (i.e., wavelength shifts to the longer wavelength side) were previously reported in two-mode fibers in Ref. 8. The insertion loss of the sensor is approximately $0 \sim$ $0.5 \mathrm{~dB}$. An extinction ratio of $5 \mathrm{~dB}$ was obtained, which depends on the power distribution between the two interfering modes. The wavelength spacing with respect to the fiber lengths and fiber core diameters as well as the polarization dependence are also investigated experimentally.

\section{Theory}

As shown in Fig. 1, both sides of the GI multimode fiber are spliced to single-mode fibers with good alignment. The fundamental mode that propagates along 


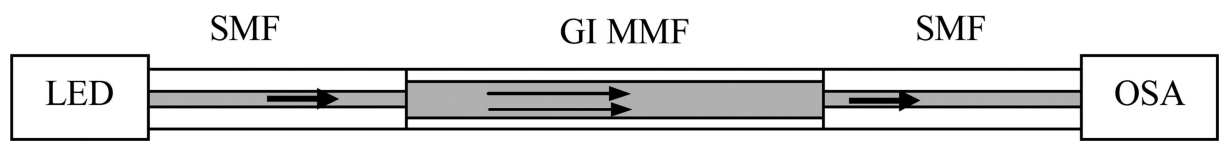

Fig. 1. Schematic of all-fiber Mach-Zehnder interferometer. SMF, single mode fiber; GI MMF, graded-index multimode fiber; LED, broadband light source; OSA, optical spectrum analyzer.

the single-mode fiber will couple into the multimode fiber, and the power will be dominantly distributed in the lowest-order mode while only a small portion of power propagates in the higher-order modes. The different modes will interfere and recouple into the single-mode fiber. When only the first two lowestorder modes are considered, the measured intensity is given by

$$
I=I_{1}+I_{2}+2 \sqrt{I_{1} I_{2}} \cos \left[\frac{2 \pi \Delta n L}{\lambda}\right]
$$

where $I_{1}$ and $I_{2}$ are the power distributed in the firstand the second-order modes $\left(I_{2} \ll I_{1}\right)$, respectively, $I$ is the measured power, $L$ is the length of the multimode fiber, $\lambda$ is the light wavelength in vacuum, $\Delta n$ $=n_{2}-n_{1}$, and $n_{1}$ and $n_{2}$ are the mode indices. The wavelength spacing $\Delta \lambda$ of the interfering spectrum is given by Eq. (1) as

$$
\Delta \lambda \approx \frac{\lambda^{2}}{\Delta n L}
$$

Clearly, $\Delta \lambda$ is inversely proportional to the length of the multimode fiber. The interfering visibility depends on the power distribution between the two interfering modes. The extinction ratio with respect to the relative power distribution $I_{2} /\left(I_{1}+I_{2}\right)$ is shown in Fig. 2; when $I_{2} /\left(I_{1}+I_{2}\right)=7 \%$, the extinction ratio is approximately $5 \mathrm{~dB}$. A high extinction ratio could be obtained by offsetting the center of the single-mode fiber core with respect to the multimode fiber, in order to obtain two modes with similar amounts of

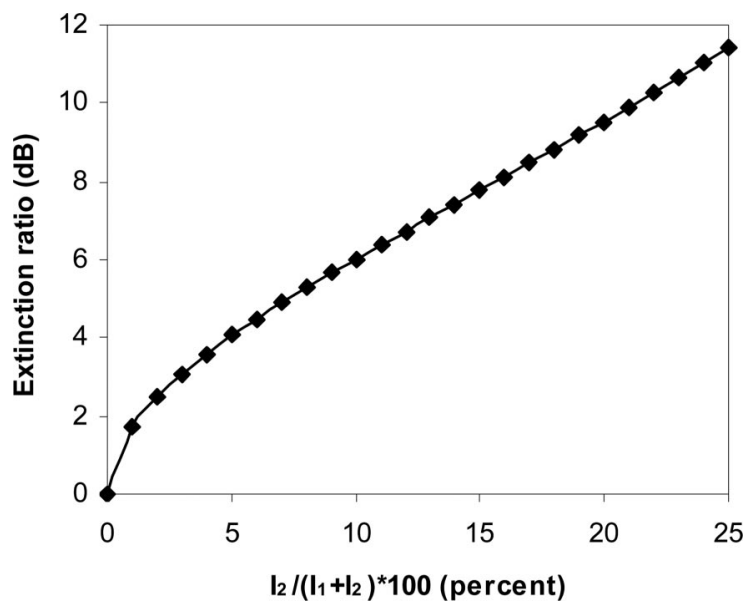

Fig. 2. Extinction ratio with respect to the relative power distribution of the two interfering modes. power to interfere with each other. However, this would introduce significant loss to the transmission.

When a strain is applied to the multimode fibers or ambient temperature changes, the mode indices and the fiber length will change, and thus the transmission peaks will shift. When the phase in Eq. (1) equals $2 N \pi,(N=1,2,3, \ldots)$ for a wavelength, the transmission reaches its peak value at this wavelength as

$$
\lambda=\Delta n L / N .
$$

By differentiating Eq. (3), we have

$$
\frac{\Delta \lambda}{\lambda} \approx\left[\frac{1}{\Delta n} \frac{\partial(\Delta n)}{\partial T}+\frac{1}{L} \frac{\partial L}{\partial T}\right] \Delta T+\left[1+\frac{1}{\Delta n} \frac{\partial(\Delta n)}{\partial \varepsilon}\right] \varepsilon,
$$

where, $\varepsilon=\mathrm{d} L / L$ indicates the applied strain. Equation (4) is very similar to the expression that describes the resonance shift of FBGs with respect to the temperature and applied strain. ${ }^{2}$ Both the changes of the temperature and the strain contribute to the wavelength shifts. We investigated the sensitivities of the temperature and the strain separately. The crosscoupling effects between the two can be studied similar to FBG sensing, which is beyond the scope of this paper.

\section{Experiment and Discussion}

As shown in Fig. 1, the GI multimode fibers with numerical apertures (NAs) of 0.275 and 0.29 for core/cladding diameters of $62.5 / 125 \mu \mathrm{m}$ and $100 /$ $140 \mu \mathrm{m}$, respectively, were used in our experiment. An Agilent 8614 optical spectrum analyzer (OSA) was used for the measurement. The OSA built-in LED and the white-light source LS-1 tungsten halogen light source were used as light sources.

For a GI multimode fiber with a length of $1.8 \mathrm{~m}$, core/cladding diameters of $100 / 140 \mu \mathrm{m}$ and NA of 0.29 , the transmission spectrum is shown in Fig. 3. The transmission loss at the peak wavelength is between 0 and $0.5 \mathrm{~dB}$. The peak wavelength spacing is $5.06 \mathrm{~nm}$, and the extinction ratio is approximately $5 \mathrm{~dB}$, corresponding to $7 \%$ of power in the second-order mode. We also investigated the transmission spectrum by using smaller diameter GI multimode fibers. Our experimental results show that the comblike peaks with larger diameter GI multimode fibers have a better extinction ratio, which is attributed to a relatively large percentage of power in the second-order mode.

The peak wavelength spacing with respect to the fiber length is shown in Fig. 4, inversely propor- 


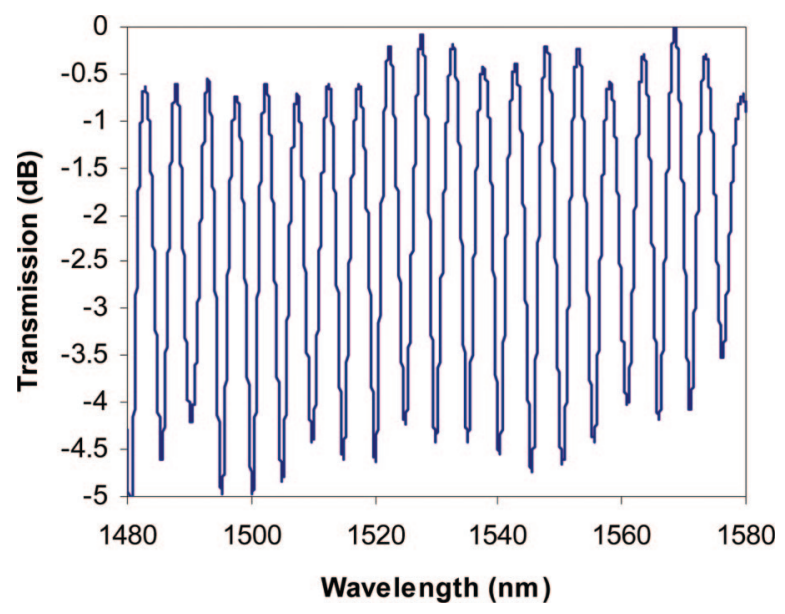

Fig. 3. Measured transmission spectrum of GI MMF, showing $0-0.5 \mathrm{~dB}$ loss at peak wavelength and $\sim 5 \mathrm{~dB}$ extinction ratio. Core/cladding diameters: $100 / 140 \mu \mathrm{m}, L=1.8 \mathrm{~m}$, NA of 0.29 .

tional to the fiber length given by Eq. (2). The dots are the experimental data for fibers with core/ cladding diameters of $62.5 / 125 \mu \mathrm{m}$, and the triangles for $100 / 140 \mu \mathrm{m}$. The slopes are $11.465 \mathrm{~m} \cdot \mathrm{m}$ and $7.028 \mathrm{~nm} \cdot \mathrm{m}$, respectively. Clearly, a smaller core diameter fiber has a relatively larger coefficient.

When a strain was applied to a GI multimode fiber, we found the comblike peaks have a blueshift with an enhanced sensitivity, as shown in Fig. 5. GI multimode fibers with core/cladding diameters of 62.5/ $125 \mu \mathrm{m}$ and $100 / 140 \mu \mathrm{m}$ were used in the experiment. The measured wavelength shifts have a good linear behavior. The strain sensitivities are 18.6 and $9.8 \mathrm{pm} /$ microstrain, respectively. It is clear that for two GI multimode fibers with close NAs, the smaller core diameter fiber has a higher strain sensitivity. This suggests that a GI multimode fiber with a small core diameter is preferred for applications in sensors. On the other hand, a large core diameter multimode fiber would be desirable for applications as a comb

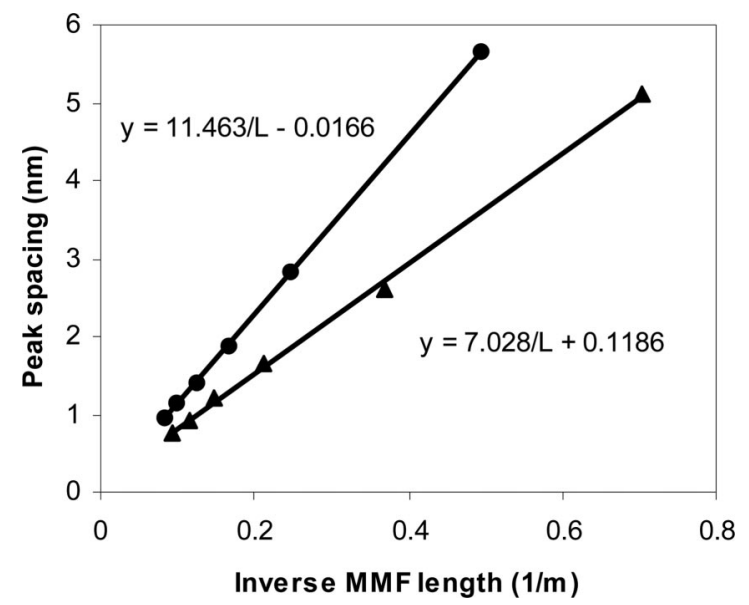

Fig. 4. Peak spacing with respect to the GI MMF fiber length, showing as inversely proportional to the fiber length. Core/ cladding diameters: 62.5/125 $\mu \mathrm{m}$ (dots); 100/140 $\mu \mathrm{m}$ (triangles).

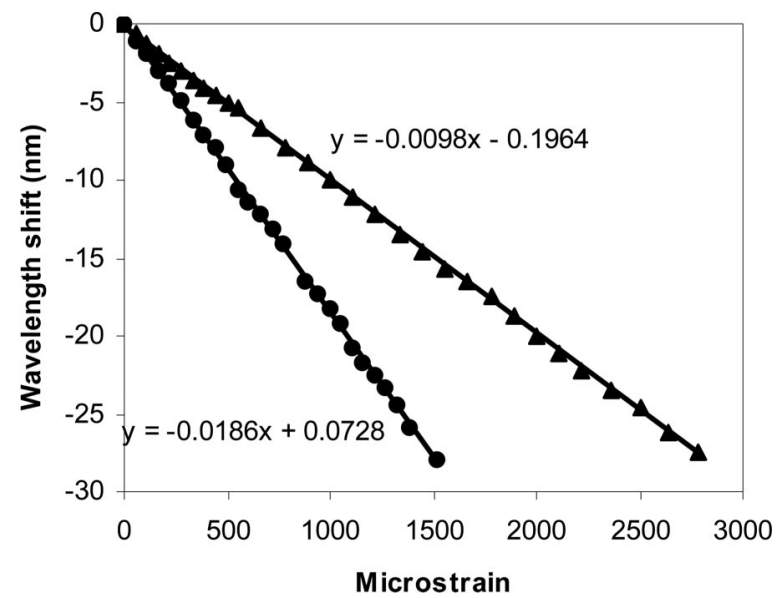

Fig. 5. Measured peak wavelength shift with respect to the applied strain, showing 18.6 and $9.8 \mathrm{pm} /$ microstrain sensitivities for the core/cladding diameters: 62.5/125 $\mu \mathrm{m}$ (dots); 100/140 $\mu \mathrm{m}$ (triangles).

filter in tunable fiber lasers owing to the large extinction ratio and less sensitivity.

The wavelength shifts were also measured with respect to the temperature. We immersed a coiled multimode fiber into a bottle of heated water. The peak wavelength shift with respect to the changes in ambient temperature is shown in Fig. 6. The dots are the experimental results, and the straight line is the fitted line. The temperature sensitivity is approximately $58.5 \mathrm{pm} /{ }^{\circ} \mathrm{C}$. Note that there is some deviation from the linearity in Fig. 6. One of the reasons is the temperature uniformity during heating and accurate temperature measurements; another factor may be the mode coupling 9 introduced by the minor change of the fiber curvature during heating.

To investigate the polarization dependence, we inserted a polarization controller into the single-mode fiber before the light was coupled to the GI multimode fiber. By tuning the polarization controller, the peak wavelengths have an approximate $0.53 \mathrm{~nm}$ shift, corresponding to $55 \%$ of peak wavelength spacing, as

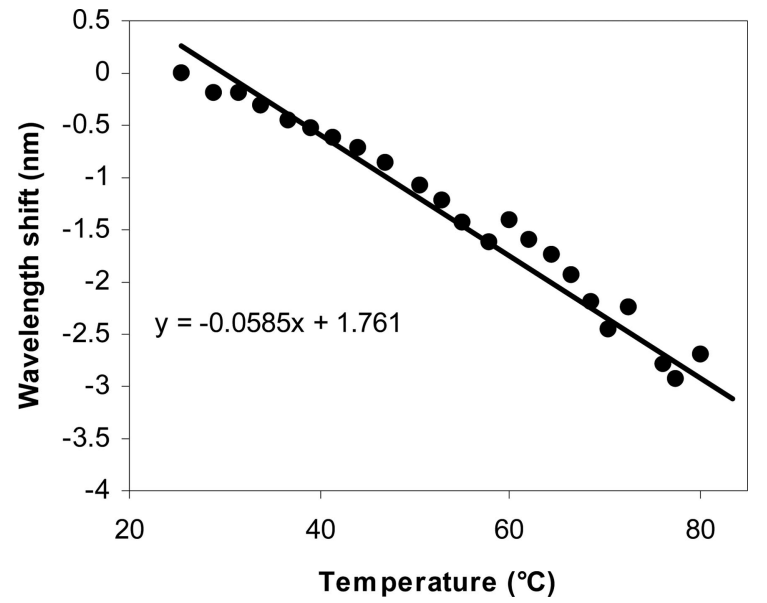

Fig. 6. Peak wavelength shift with respect to the changes of ambient temperature, showing a $58.5 \mathrm{pm} /{ }^{\circ} \mathrm{C}$ sensitivity. 


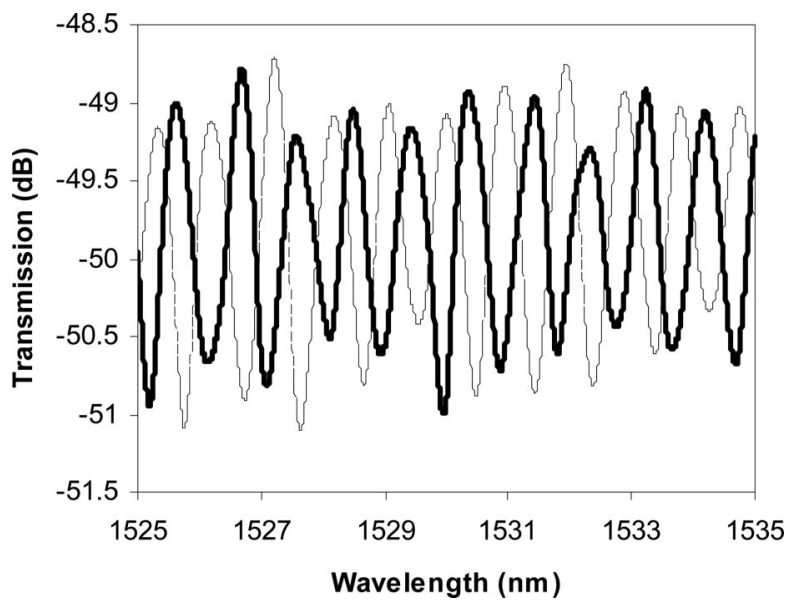

Fig. 7. Polarization dependence of GI multimode fiber-based MZI, showing $55 \%$ of wavelength spacing $(0.53 \mathrm{~nm})$ polarization shifts.

shown in Fig. 7. This feature may be used for tuning fiber laser wavelengths when a GI multimode fiber is used as a comb filter.

\section{Conclusion}

A simple and high-sensitivity temperature and strain sensor based on mode interference in graded-index multimode fibers has been reported. The strain and temperature characterizations are presented. Blueshifts with enhanced sensitivities of $18.6 \mathrm{pm} /$ microstrain and $58.5 \mathrm{pm} /{ }^{\circ} \mathrm{C}$ were observed. Smaller diameter core fibers have significantly larger strain sensitivity. We also investigated the polarization dependence and found $0.53 \mathrm{~nm}$ that corresponds to $55 \%$ of wavelength spacing can be tuned by using a polarization controller. Such an interfering effect in graded-index multimode fiber should find useful applications in fiber sensing and tunable multiwavelength fiber lasers for instrumentations and telecommunications.

This work was supported by the Natural Sciences and Engineering Research Council of Canada (NSERC).

\section{References}

1. J. Yuan and M. A. El-Sherif, "Fiber-optic chemical sensor using polyaniline as modified cladding material," IEEE Sens. J. 3, 5-12 (2003).

2. A. D. Kersey, M. A. Davis, H. J. Patrick, M. LeBlanc, K. P. Koo, C. G. Askins, M. A. Putnam, and E. J. Friebele, "Fiber grating sensors," J. Lightwave Technol. 15, 1442-1463 (1997).

3. Y. C. Han, S. B. Lee, C. S. Kim, J. U. Kang, U. C. Paek, and Y. Chuang, "Simultaneous measurement of temperature and strain using dual long-period fiber gratins with controlled temperature and strain sensitivities," Opt. Exp. 11, 476-481 (2003).

4. A. Kumar, N. K. Goel, and R. K. Varshney, "Studies on a fewmode fiber-optic strain sensor based on $\mathrm{LP}_{01}-\mathrm{LP}_{02}$ mode interference," J. Lightwave Technol. 19, 358-362 (2001).

5. Q. Wang and G. Farrell, "All-fiber multimode-interferencebased refractometer sensor: proposal and design," Opt. Lett. 31, 317-319 (2006)

6. A. Mehta, W. Mohammed, and E. G. Johnson, "Multimode interference-based fiber-optic displacement sensor," IEEE Photon. Technol. Lett. 15, 1129-1131 (2003).

7. T. J. Chen, "Use of liquid-crystal-clad fiber as a modal filter for a two-mode fiber-optic interferometer," Opt. Lett. 29, 28522854 (2004).

8. Q. Li, C. H. Lin, P. Y. Tseng, and H. P. Lee, "Demonstration of high extinction ratio modal interference in a two-mode fiber and its applications for all-fiber comb filter and high-temperature sensor," Opt. Commun. 250, 280-285 (2005).

9. S. Savovic and A. Djordjevich, "Mode coupling in strained and unstrained step-index plastic optical fibers," Appl. Opt. 45, 6775-6780 (2006) 